\title{
Discretization of Homogeneous Systems Using Euler Method with a State-Dependent Step
}

\author{
Denis Efimov $^{\mathrm{a}, \mathrm{b}}$, Andrey Polyakov ${ }^{\mathrm{a}, \mathrm{b}}$, Alexander Aleksandrov ${ }^{\mathrm{c}}$ \\ anria, Univ. Lille, CNRS, UMR 9189 - CRIStAL, F-59000 Lille, France \\ ${ }^{\mathrm{b}}$ ITMO University, 49 av. Kronverkskiy, 197101 Saint Petersburg, Russia \\ ${ }^{\mathrm{c}}$ Saint Petersburg State University, 7-9 Universitetskaya nab., 199034 Saint Petersburg, Russia
}

\begin{abstract}
Numeric approximations to the solutions of asymptotically stable homogeneous systems by Euler method, with a step of discretization scaled by the state norm, are investigated (for the explicit and implicit integration schemes). It is proven that for a sufficiently small discretization step the convergence of the approximating solutions to zero can be guaranteed globally in a finite or a fixed time depending on the degree of homogeneity of the system, but in an infinite number of discretization iterations. The maximal admissible step can be estimated by analyzing the system properties on the sphere. It is shown that the absolute and relative errors of the discretizations are globally bounded functions, thus the approximations approaching the solutions with the step converging to zero. In addition, it is established that the proposed discretization approach preserves robustness with respect to exogenous perturbations. Efficiency of the designed discretization algorithms is demonstrated in simulations.
\end{abstract}

Key words: Homogeneous systems; Discretization; Euler method.

\section{Introduction}

In many applications non-asymptotic convergence rates are preferable, and the theory of homogeneous dynamical systems are frequently used to guarantee these fast rates [7]. Examples of such an application of homogeneity can be found in the sliding mode control domain [28], in the designs seeking for control or estimation in finite-time $[23,24,11,35,31,41,42]$ or in fixed-time $[5,14,37,38]$. Another reason of popularity of homogeneous systems is due to that they take an intermediate place between linear and nonlinear dynamics [6] (the linear dynamical systems are also homogeneous). The common advantages of homogeneous systems include scalability of trajectories, robustness to measurement noises and exogenous disturbances $[2,36,9]$, e.g. as the linear dynamics. From another side, these systems are

\footnotetext{
^ This work was partially supported by ANR 18 CE40-0008 (Project DIGITSLID), the Government of Russian Federation (Grant 08-08) and Saint Petersburg State University (Project Id 37569826).

Email addresses: denis.efimov@inria.fr (Denis Efimov), andrey.polyakov@inria.fr (Andrey Polyakov), a.u.aleksandrov@spbu.ru (Alexander Aleksandrov).
}

described by essentially nonlinear differential equations, where some interesting nonlinear phenomena can be observed, namely finite- or fixed-time convergence rates for negative and positive degrees of homogeneity, respectively, and robustness with respect to delays [19,43].

The models of industrial plants are usually described by continuous-time ordinary differential equations, then frequently the control and estimation algorithms are developed in continuous-time setting. Hence, design and analysis of stability or performance of closed-loop systems are performed in continuous time, while for a verification or implementation, the solutions of closed-loop systems have to be calculated in a computer or in a digital controller, which are usually operating in a discrete time. Different numerical discrete-time approximation methods and time discretization schemes are proposed for continuous-time differential equations in order to realize such an implementation $[4,13]$. Their variety is originated by the fact that these approaches guarantee the preservation of performances obtained in continuous time for discrete-time approximations only under some particular hypothesis, then their application is case-dependent. For the control or estimation algorithms designed using the theory of homogeneous sy- 
stems, which possess an increased rate of convergence with respect to linear systems, the problem of implementation and derivation of solutions becomes important being in the focus of many works, especially in the sliding mode control community $[29,18]$.

The conventional Euler method is a first-order numerical routine for solving ordinary differential equations with a given initial value and a fixed time step, which represents the most basic explicit/implicit method of numerical integration and the simplest Runge-Kutta method, which is extremely popular in the control community. In particular, in the recent papers $[18,30]$ it has been shown that application of the explicit Euler method (with a fixed time step) for the global approximation of solutions of homogeneous systems with non-zero degree is problematic (see also [29]), and the implicit Euler scheme has a better perspective (as it has been also indicated in $[1,12,25,34])$. In other papers, the conditions of convergence and stability of the explicit and implicit Euler methods have been studied for linear systems (the notion of A-stability) [16,13], or for particular classes of nonlinear systems $[22,33,3]$.

Following the results obtained in [18], the present work is devoted to application of explicit and implicit Euler discretization schemes for approximation of solutions of homogeneous stable dynamical systems. The case of a state-dependent scaling of the time discretization step is considered, and different conditions for the existence (in the implicit case) and convergence to zero of approximations for the explicit and implicit Euler integration methods are derived. It is shown that introduction of a state-dependent re-scaling of the time discretization step allows the finite- or fixed-time rates of convergence to be recovered by the discrete-time approximations of solutions, but for an infinite number of steps (in a finite number of steps the convergence to a vicinity of the origin is obtained). Absolute and relative errors (closeness of the approximations to real solutions) for the explicit and implicit Euler integration schemes are investigated using the homogeneity theory, and it is shown that the relative errors are globally bounded and by decreasing the initial discretization step it is possible to make them arbitrary small, hence, the proposed modification of the Euler method can be indeed used for calculation of solutions of homogeneous dynamics. The preliminary formulation of these results (without proofs) appears in [17]. In the present work, the proofs are given and, in addition, since stable homogeneous systems admit certain robustness with respect to external inputs [9], it is shown that the discretized approximations obtained with the proposed methods keep this property.

The outline of this note is as follows. The problem statement and some preliminary results are introduced in Section 2. Some basic properties and relations between solution approximations are studied in Section 3. The convergence conditions are established in Section 4. Pro- perties of relative and absolute errors of approximation of solutions of homogeneous systems by the explicit and implicit Euler methods are investigated in Section 5. Analysis of stability robustness of approximations with respect to external disturbances is presented in Section 6. Simple illustrating example is considered in Section 7.

\section{Notation}

- $\mathbb{R}_{+}=\{x \in \mathbb{R}: x \geq 0\}$, where $\mathbb{R}$ is the set of real number.

- $|\cdot|$ denotes the absolute value in $\mathbb{R}$, for a vector $x \in \mathbb{R}^{n}$ the symbol $\|x\|$ denotes the Euclidean norm, and the corresponding induced matrix norm $\|A\|$ for a matrix $A \in \mathbb{R}^{n \times n}$.

- A continuous function $\alpha: \mathbb{R}_{+} \rightarrow \mathbb{R}_{+}$belongs to the class $\mathcal{K}$ if $\alpha(0)=0$ and the function is strictly increasing. The function $\alpha: \mathbb{R}_{+} \rightarrow \mathbb{R}_{+}$belongs to the class $\mathcal{K}_{\infty}$ if $\alpha \in \mathcal{K}$ and it is increasing to infinity.

- The identity matrix of dimension $n \times n$ is denoted as $I_{n}$, and $\operatorname{diag}\left\{r_{i}\right\}_{i=1}^{n}$ is a diagonal matrix with the elements on the main diagonal equal $r_{i}$.

- A sequence of integers $1,2, \ldots, n$ is denoted by $\overline{1, n}$.

\section{$2 \quad$ Preliminaries}

In this work the following nonlinear system is considered:

$$
\dot{x}(t)=f(x(t)), t \geq 0,
$$

where $x(t) \in \mathbb{R}^{n}$ is the state, $f: \mathbb{R}^{n} \rightarrow \mathbb{R}^{n}$ ensures forward existence and uniqueness of the system solutions at least locally, $f(0)=0$. For an initial condition $x_{0} \in \mathbb{R}^{n}$ define the corresponding solution by $X\left(t, x_{0}\right)$ for any $t \geq 0$ for which the solution exists. If $f$ is discontinuous, then the solutions are understood in the Filippov's sense [21] (it is assumed that $f(x) \in \Phi(x)$, where $\Phi$ is the corresponding Filippov's set-valued extension).

Following $[40,27,37]$, let $\Omega \subset \mathbb{R}^{n}$ be an open set, $0 \in \Omega$.

Definition 1 At the steady state $x=0$ the system (1) is said to be

(a) stable if for any $x_{0} \in \Omega$ the solution $X\left(t, x_{0}\right)$ is defined for all $t \geq 0$, and for any $\epsilon>0$ there is $\delta>0$ such that for any $x_{0} \in \Omega$, if $\left\|x_{0}\right\| \leq \delta$ then $\left\|X\left(t, x_{0}\right)\right\| \leq \epsilon$ for all $t \geq 0$;

(b) asymptotically stable if it is stable and for any $\kappa>0$ and $\epsilon>0$ there exists $T(\kappa, \epsilon) \geq 0$ such that for any $x_{0} \in \Omega$, if $\left\|x_{0}\right\| \leq \kappa$ then $\left\|X\left(t, x_{0}\right)\right\| \leq \epsilon$ for all $t \geq T(\kappa, \epsilon)$;

(c) finite-time stable if it is stable and finite-time converging from $\Omega$, i.e. for any $x_{0} \in \Omega$ there exists $0 \leq T<$ $+\infty$ such that $X\left(t, x_{0}\right)=0$ for all $t \geq T$. The function 
$T_{0}\left(x_{0}\right)=\inf \left\{T \geq 0: X\left(t, x_{0}\right)=0 \forall t \geq T\right\}$ is called the settling time of the system (1);

(d) fixed-time stable if it is finite-time stable and $\sup _{x_{0} \in \Omega} T_{0}\left(x_{0}\right)<+\infty$.

The set $\Omega$ is called a domain of stability/attraction.

If $\Omega=\mathbb{R}^{n}$, then the corresponding properties are called global stability/asymptotic stability/finite-time/fixedtime stability of (1) at $x=0$.

Similarly, the stability notions can be defined with respect to a compact invariant set, by replacing the distance to the origin in Definition 1 with the distance to a set.

\subsection{Weighted homogeneity}

Following $[44,6,28]$, for strictly positive numbers $r_{i}, i=$ $\overline{1, n}$ called weights and $\lambda>0$, define:

- the vector of weights $\mathbf{r}=\left(r_{1}, \ldots, r_{n}\right)^{\top}, r_{\max }=$ $\max _{1 \leq j \leq n} r_{j}$ and $r_{\min }=\min _{1 \leq j \leq n} r_{j}$

- the dilation matrix function $\Lambda_{r}(\lambda)=\operatorname{diag}\left\{\lambda^{r_{i}}\right\}_{i=1}^{n}$, note that $\forall x \in \mathbb{R}^{n}$ and $\forall \lambda>0$ we have $\Lambda_{r}(\lambda) x=$ $\left(\lambda^{r_{1}} x_{1}, \ldots, \lambda^{r_{n}} x_{n}\right)^{\top}$

- the $\mathbf{r}$-homogeneous norm $\|x\|_{r}=\left(\sum_{i=1}^{n}\left|x_{i}\right|^{\frac{\rho}{r_{i}}}\right)^{\frac{1}{\rho}}$ for any $x \in \mathbb{R}^{n}$ and $\rho \geq r_{\max }$, it is not a norm in the standard sense, since the triangle inequality is not satisfied for $\|\cdot\|_{r}$, however there exist $\underline{\sigma}, \bar{\sigma} \in \mathcal{K}_{\infty}$ such that

$$
\underline{\sigma}\left(\|x\|_{r}\right) \leq\|x\| \leq \bar{\sigma}\left(\|x\|_{r}\right) \quad \forall x \in \mathbb{R}^{n}
$$

- the sphere and the ball in the homogeneous norm $S_{r}(\varrho)=\left\{x \in \mathbb{R}^{n}:\|x\|_{r}=\varrho\right\}$ and $B_{r}(\varrho)=\left\{x \in \mathbb{R}^{n}\right.$ : $\left.\|x\|_{r} \leq \varrho\right\}$ for $\varrho \geq 0$.

Definition 2 A function $g: \mathbb{R}^{n} \rightarrow \mathbb{R}$ is $\mathbf{r}$-homogeneous with degree $\mu \in \mathbb{R}$ if $\forall x \in \mathbb{R}^{n}$ and $\forall \lambda>0$ we have:

$$
\lambda^{-\mu} g\left(\Lambda_{r}(\lambda) x\right)=g(x)
$$

$A$ vector field $f: \mathbb{R}^{n} \rightarrow \mathbb{R}^{n}$ is $\mathbf{r}$-homogeneous with degree $\nu \in \mathbb{R}, \nu \geq-r_{\min }$ if $\forall x \in \mathbb{R}^{n}$ and $\forall \lambda>0$ we have:

$$
\lambda^{-\nu} \Lambda_{r}^{-1}(\lambda) f\left(\Lambda_{r}(\lambda) x\right)=f(x)
$$

which is equivalent for $i$-th component of $f$ being a $\mathbf{r}$-homogeneous function of degree $r_{i}+\nu$.

System (1) is $\mathbf{r}$-homogeneous of degree $\nu$ if the vector field $f$ is $\mathbf{r}$-homogeneous of the degree $\nu$.

The homogeneous norm $\|\cdot\|_{r}$ is an example of $\mathbf{r}$-homogeneous function with degree 1 .
Theorem 3 [44,39] For the system (1) with $\mathbf{r}$-homogeneous and continuous function $f$ the following properties are equivalent:

- the system (1) is asymptotically stable;

- there exists a $C^{2} \mathbf{r}$-homogeneous Lyapunov function $V: \mathbb{R}^{n} \rightarrow \mathbb{R}_{+}$such that $\forall x \in \mathbb{R}^{n}$ and $\forall \lambda>0$

$$
\begin{gathered}
\alpha_{1}(\|x\|) \leq V(x) \leq \alpha_{2}(\|x\|), \\
L_{f} V(x)=\frac{\partial V}{\partial x}(x) f(x) \leq-\alpha(\|x\|), \\
\lambda^{-\mu} V\left(\Lambda_{r}(\lambda) x\right)=V(x), \mu>r_{\max },
\end{gathered}
$$

for some $\alpha_{1}, \alpha_{2} \in \mathcal{K}_{\infty}$ and $\alpha \in \mathcal{K}$.

The requirement on continuity of the function $f$ has been relaxed in [8].

\section{$3 \quad$ Euler schemes}

Let us consider a class of systems in (1) with

$$
\sup _{\xi \in S_{r}(1)}\|f(\xi)\|<+\infty
$$

and admitting the following hypothesis:

Assumption 1 Let (1) be $\mathbf{r}$-homogeneous with a degree $\nu \in \mathbb{R}$ and asymptotically stable.

In this work, in order to obtain a discretization of the solutions of (1), we will propose modifications of implicit and explicit Euler methods [13] with state-dependent scaling of the time discretization step. To this end, select a basic discretization step $h>0$, define a sequence of time instants $t_{i}$ for $i=0,1, \ldots$ such that $t_{0}=0$ and $t_{i+1}-t_{i}>0$, and denote by $x_{i}$ an approximation of the solution $X\left(t_{i}, x_{0}\right)$ at the corresponding time instant (i.e. $x_{i} \simeq X\left(t_{i}, x_{0}\right)$ and $\left.x_{0}=X\left(0, x_{0}\right)\right)$, then the approximation $x_{i+1}$ calculated in accordance with the explicit Euler method is given by:

$$
\begin{aligned}
x_{i+1} & =x_{i}+\frac{h}{\left\|x_{i}\right\|_{r}^{\nu}} f\left(x_{i}\right), \\
t_{i+1} & =t_{i}+\frac{h}{\left\|x_{i}\right\|_{r}^{\nu}}
\end{aligned}
$$

for $i=0,1, \ldots$, while the approximation calculated by the implicit Euler method comes from:

$$
\begin{aligned}
x_{i+1} & =x_{i}+\frac{h}{\left\|x_{i+1}\right\|_{r}^{\nu}} f\left(x_{i+1}\right), \\
t_{i+1} & =t_{i}+\frac{h}{\left\|x_{i+1}\right\|_{r}^{\nu}}
\end{aligned}
$$

for $i=0,1, \ldots$ In the algorithms (2) and (3) it is assumed that $x_{i} \neq 0$ or $x_{i+1} \neq 0$ since in the opposite 
case the discretization stops at the equilibrium due to $f(0)=0$.

Remark 4 In order to clarify the relations of the results obtained in this work and in [18], and also to motivate the selected design, for the system (1) under Assumption 1 let us consider a function

$$
\psi_{x_{0}}(t)=\int_{0}^{t}\left\|X\left(s, x_{0}\right)\right\|_{r}^{\nu} d s
$$

for any $x_{0} \in \mathbb{R}^{n}$, which is well-defined and invertible when the trajectory stays out of the origin, i.e. for all $t \in\left[0, T_{0}\left(x_{0}\right)\right)$ where $T_{0}: \mathbb{R}^{n} \rightarrow \mathbb{R}_{+} \cup\{+\infty\}$ is possibly infinite time of convergence to the origin (as in Definition 1). Denote $Y\left(\tau, x_{0}\right)=X\left(\psi_{x_{0}}^{-1}(\tau), x_{0}\right)$ for $\tau \in$ $\left[0, \psi_{x_{0}}\left(T_{0}\left(x_{0}\right)\right)\right)$, then

$$
\begin{gathered}
\frac{d}{d \tau} Y\left(\tau, x_{0}\right)=\left.\frac{d}{d s} X\left(s, x_{0}\right)\right|_{s=\psi_{x_{0}}^{-1}(\tau)} \frac{1}{\left\|X\left(\psi_{x_{0}}^{-1}(\tau), x_{0}\right)\right\|_{r}^{\nu}} \\
=\frac{f\left(X\left(\psi_{x_{0}}^{-1}(\tau), x_{0}\right)\right)}{\left\|X\left(\psi_{x_{0}}^{-1}(\tau), x_{0}\right)\right\|_{r}^{\nu}}=\frac{f\left(Y\left(\tau, x_{0}\right)\right)}{\left\|Y\left(\tau, x_{0}\right)\right\|_{r}^{\nu}}
\end{gathered}
$$

Obviously, for any $x_{0} \in \mathbb{R}^{n}$ the asymptotic stability properties of the corresponding solutions $X\left(t, x_{0}\right)$ of the system (1) for $t \in\left[0, T_{0}\left(x_{0}\right)\right)$ and $Y\left(\tau, x_{0}\right)$ of (4) for $\tau \in$ $\left[0, \psi_{x_{0}}\left(T_{0}\left(x_{0}\right)\right)\right)$ are interrelated, and the system (4) is $\mathbf{r}$-homogeneous with degree 0 :

$$
\frac{f\left(\Lambda_{r}(\lambda) y\right)}{\left\|\Lambda_{r}(\lambda) y\right\|_{r}^{\nu}}=\Lambda_{r}(\lambda) \frac{f(y)}{\|y\|_{r}^{\nu}}
$$

for any $y \in \mathbb{R}^{n}$ and $\lambda>0$. As it has been shown in [18], for the case $\nu=0$ and a properly established discretization step $h>0$ the explicit and implicit Euler methods provide an admissible approximation of the solution $y_{i} \simeq Y\left(\tau_{i}, x_{0}\right)$ with $\tau_{i}=$ ih for $i=0,1, \ldots$ :

$$
y_{i+1}=y_{i}+\frac{h}{\left\|y_{i}\right\|_{r}^{\nu}} f\left(y_{i}\right), y_{i+1}=y_{i}+\frac{h}{\left\|y_{i+1}\right\|_{r}^{\nu}} f\left(y_{i+1}\right) .
$$

Hence,

$$
\tau_{i+1}-\tau_{i}=h=\int_{t_{i}}^{t_{i+1}}\left\|X\left(s, x_{0}\right)\right\|_{r}^{\nu} d s
$$

where $t_{i}$ are the corresponding instants of discretization in the original time $t$, and under an assumption that $\left\|X\left(s, x_{0}\right)\right\|_{r}^{\nu} \simeq$ const for $s \in\left[t_{i}, t_{i+1}\right]$, i.e. the discret $i$ zation interval is sufficiently small relative to the velocity of the system, we obtain $t_{i+1}-t_{i} \simeq \frac{h}{\left\|X\left(t_{i}, x_{0}\right)\right\|_{r}^{\nu}}$ or $t_{i+1}-t_{i} \simeq \frac{h}{\left\|X\left(t_{i+1}, x_{0}\right)\right\|_{r}^{\nu}}$ for (2) and (3), respectively. In this work such a scheme is used directly for the system (1) to approximate the solution $X\left(t_{i}, x_{0}\right)$, which is a significant difference from [18]. This intuition also highlights our motivation to reduce an Euler computation scheme to work with a system of degree zero.

Therefore, it is a well-known fact that for $\nu=0$ with $h \rightarrow 0$ both methods approach the real solution [13], i.e. $x_{i} \rightarrow X\left(t_{i}, x_{0}\right)$ in $(2)$ and (3) with $h \rightarrow 0$ over any compact time interval. In [18] for the case of the conventional explicit and implicit Euler methods with a constant discretization step $h$ and $\nu \neq 0$ it has been shown that outside of a vicinity of the origin for $\nu<0$ or a vicinity of infinity for $\nu>0$ the same results can be obtained.

In the sequel, the problem of global convergence to zero of the approximations $\left\{x_{i}\right\}_{i=0}^{\infty}$ derived in (2) and (3) is studied for systems in (1) satisfying Assumption 1 with $\nu \neq 0$ (the case $\nu=0$, or without scaling of the discretization step, has been analyzed in [18]). For this goal we need to establish some auxiliary properties of approximations $\left\{x_{i}\right\}_{i=0}^{\infty}$ obtained in (2) and (3).

\subsection{Relations between approximations obtained for dif- ferent initial conditions}

One of the main features of (2) and (3) is as follows:

Proposition 5 Let the system (1) be $\mathbf{r}$-homogeneous with a degree $\nu \in \mathbb{R}$. If $\left\{x_{i}\right\}_{i=0}^{\infty}$ is a sequence generated by (2) or (3) for the time instants $\left\{t_{i}\right\}_{i=0}^{\infty}$ with the step $h$ and the initial state $x_{0}$, then for any $\lambda>0, y_{i}=\Lambda_{r}(\lambda) x_{i}$ is a sequence obtained by (2) or (3), respectively, for the instants $\lambda^{-\nu}\left\{t_{i}\right\}_{i=0}^{\infty}$ with the step $h$ and the initial state $y_{0}=\Lambda_{r}(\lambda) x_{0}$.

PROOF. Fixing $\lambda>0$ and multiplying both sides of (2) by the dilation matrix $\Lambda_{r}(\lambda)$ we obtain:

$$
\begin{aligned}
\Lambda_{r}(\lambda) x_{i+1} & =\Lambda_{r}(\lambda) x_{i}+\frac{h}{\lambda^{\nu}\left\|x_{i}\right\|_{r}^{\nu}} \lambda^{\nu} \Lambda_{r}(\lambda) f\left(x_{i}\right) \\
& =\Lambda_{r}(\lambda) x_{i}+\frac{h}{\left\|\Lambda_{r}(\lambda) x_{i}\right\|_{r}^{\nu}} f\left(\Lambda_{r}(\lambda) x_{i}\right)
\end{aligned}
$$

or, equivalently, $y_{i+1}=y_{i}+\frac{h}{\left\|y_{i}\right\|_{r}^{\nu}} f\left(y_{i}\right)$ that gives the desired result. The proof for the scheme (3) is the same.

Note that $y_{i}$ is an approximation of $X\left(\lambda^{-\nu} t_{i}, y_{0}\right)$ for scaled instants of time.

Corollary 6 Let the system (1) be $\mathbf{r}$-homogeneous with a degree $\nu \in \mathbb{R}$. Let for all $x_{0} \in S_{r}(1)$ there exist sequences $\left\{x_{i}\right\}_{i=0}^{\infty}$ obtained by (2) or (3) with the step $h>0$ and the initial state $x_{0}$ possessing one of the properties:

$$
\begin{gathered}
\sup _{i \geq 0}\left\|x_{i}\right\|<+\infty \\
\lim _{i \rightarrow+\infty} x_{i}=0 .
\end{gathered}
$$


Then for any $y_{0} \in \mathbb{R}^{n}$ there exist sequences $\left\{y_{i}\right\}_{i=0}^{\infty}$ generated by (2) or (3) with the step $h$ and the initial state $y_{0}$ possessing the same property.

PROOF. For any $y_{0} \in \mathbb{R}^{n}$ there exists $\lambda>0$ such that $y_{0}=\Lambda_{r}(\lambda) x_{0}$ for some $x_{0} \in S_{r}(1)$, next the result is a direct consequence of Proposition 5 since $y_{i}=\Lambda_{r}(\lambda) x_{i}$.

\subsection{Norm deviations}

Another property of the proposed approximation algorithms (2) and (3) consists in their finite increment:

Lemma 7 Let the system (1) be $\mathbf{r}$-homogeneous with a degree $\nu \in \mathbb{R}$. Then there are $h_{0}>0$ and $0<\underline{c}<\bar{c}<+\infty$ such that for any $h \in\left(0, h_{0}\right]$ :

$$
\underline{c}\left\|x_{i}\right\|_{r} \leq\left\|x_{i+1}\right\|_{r} \leq \bar{c}\left\|x_{i}\right\|_{r}
$$

for all $x_{i} \in \mathbb{R}^{n}$ in (2) or (3) (provided that a solution $x_{i+1}$ exists).

PROOF. Starting with (2), let $x_{i} \in \mathbb{R}^{n}$ and denote $x_{i}=\Lambda_{r}\left(\left\|x_{i}\right\|_{r}\right) \xi_{i}$ for some $\xi_{i} \in S_{r}(1)$, then the equation (2) can be rewritten as follows:

$$
x_{i+1}=\Lambda_{r}\left(\left\|x_{i}\right\|_{r}\right)\left(\xi_{i}+h f\left(\xi_{i}\right)\right) .
$$

Therefore, $\left\|x_{i+1}\right\|_{r}=\left\|x_{i}\right\|_{r}\left\|\xi_{i}+h f\left(\xi_{i}\right)\right\|_{r}$ and there exists $h_{0}>0$ such that $0<\underline{c}<\bar{c}<+\infty$ for

$$
\begin{aligned}
& \underline{c}=\inf _{h \in\left(0, h_{0}\right]} \inf _{\xi \in S_{r}(1)}\|\xi+h f(\xi)\|_{r}, \\
& \bar{c}=\sup _{h \in\left(0, h_{0}\right]} \sup _{\xi \in S_{r}(1)}\|\xi+h f(\xi)\|_{r}
\end{aligned}
$$

since $\|f(\xi)\|$ is bounded for any $\xi \in S_{r}(1)$.

For $(3)$, let $x_{i} \in \mathbb{R}^{n}$ and denote $x_{i+1}=\Lambda_{r}\left(\left\|x_{i+1}\right\|_{r}\right) \xi_{i+1}$ for some $\xi_{i+1} \in S_{r}(1)$, then the equation (3) can be rewritten:

$$
x_{i}=\Lambda_{r}\left(\left\|x_{i+1}\right\|_{r}\right)\left(\xi_{i+1}-h f\left(\xi_{i+1}\right)\right),
$$

and the statement follows repeating the steps as for (2).

Corollary $\mathbf{8}$ Let the system (1) be $\mathbf{r}$-homogeneous with a degree $\nu \in \mathbb{R}$. Select $h_{0}>0$, then there exists $0<c<$ $+\infty$ such that for any $h \in\left(0, h_{0}\right]$ and for all $x_{i} \in \mathbb{R}^{n}$, $\left\|x_{i+1}\right\|_{r} \leq c\left\|x_{i}\right\|_{r}$ in (2) or $c\left\|x_{i}\right\|_{r} \leq\left\|x_{i+1}\right\|_{r}$ in (3) (provided that a solution $x_{i+1}$ exists).

PROOF. This result follows repeating the arguments of the proof of Lemma 7 by taking into account that for a generic $f$ it might be $\inf _{h \in\left(0, h_{0}\right]} \inf _{\xi \in S_{r}(1)} \| \xi+$ $h f(\xi)\left\|_{r}=\inf _{h \in\left(0, h_{0}\right]} \inf _{\xi \in S_{r}(1)}\right\| \xi-h f(\xi) \|_{r}=0$ without a restriction on the amplitude of $h_{0}$.

\section{Convergence of sequences $\left\{x_{i}\right\}_{i=0}^{\infty}$ generated by Euler methods}

In this section we will study the stability and convergence features of $\left\{x_{i}\right\}_{i=0}^{\infty}$ only. The quality of the corresponding approximations (the closeness of $\left\{x_{i}\right\}_{i=0}^{\infty}$ to the continuous-time solutions $\left.X\left(t_{i}, x_{0}\right)\right)$ will be considered in the next section.

According to Theorem 3, under Assumption 1 for the system (1) there is a twice continuously differentiable and $\mathbf{r}$-homogeneous Lyapunov function $V: \mathbb{R}^{n} \rightarrow \mathbb{R}_{+}$ of the degree $\mu>-\nu$ such that

$$
\begin{gathered}
a=-\sup _{\xi \in S_{r}(1)} L_{f} V(\xi)>0, \\
0<b=\sup _{\xi \in B_{r}(1)}\left\|\frac{\partial V(\xi)}{\partial \xi}\right\|<+\infty, \\
0<c_{1}=\inf _{\xi \in S_{r}(1)} V(\xi), 0<c_{2}=\sup _{\xi \in S_{r}(1)} V(\xi), \\
c_{1}\|x\|_{r}^{\mu} \leq V(x) \leq c_{2}\|x\|_{r}^{\mu} \quad \forall x \in \mathbb{R}^{n} .
\end{gathered}
$$

\subsection{Convergence of the explicit Euler scheme (2)}

The principal statement of this subsection is as follows:

Theorem 9 Let Assumption 1 be satisfied, then there exists $h_{0}>0$ such that for any discretization step $h \in$ $\left(0, h_{0}\right]$ the sequences $\left\{x_{i}\right\}_{i=0}^{\infty}$ obtained by (2) for any initial state $x_{0} \in \mathbb{R}^{n}$ and the step $h$ possess the following properties:

(a) $\sup _{i=0,1, \ldots}\left\|x_{i}\right\|_{r}<\gamma\left\|x_{0}\right\|_{r}$ for some $\gamma \in(0,+\infty)$;

(b) $\lim _{i \rightarrow+\infty}\left\|x_{i}\right\|_{r}=0$;

(c) for $\nu=0$ the sequence $\left\{x_{i}\right\}_{i=0}^{\infty}$ has an exponential convergence rate, for $\nu<0$ for any $x_{0} \in \mathbb{R}^{n}$ the time of convergence to the origin $t_{+\infty}^{x_{0}}=\lim _{i \rightarrow+\infty} t_{i}$ is finite, and for $\nu>0$ the time of convergence from any $x_{0} \in \mathbb{R}^{n}$ to $B_{r}(1)$ is also finite independently of $x_{0}$.

PROOF. The proof is given in [20].

The property obtained for $\nu>0$ is related with the fixed-time rate of convergence (to the unit ball). 
Remark 10 The maximal admissible discretization step $h_{0}$ can be evaluated as

$$
h_{0}=\min \left\{1, \frac{a}{v}, 2 \frac{c_{2}}{a}\right\}
$$

where

$$
\begin{gathered}
a=\inf _{y \in S_{r}(1)} \frac{\partial V(y)}{\partial y} f(y), c_{2}=\sup _{y \in S_{r}(1)} V(y), \\
v=\left.\sup _{y \in S_{r}(1)} \sup _{\theta \in(0,1)} f^{\top}(y) \frac{\partial^{2} V(\xi)}{\partial \xi^{2}}\right|_{\xi=y+\theta f(y)} f(y)
\end{gathered}
$$

are calculated on the unit sphere for known $f$ and $V$. Such an estimate is a lower bound on $h_{0}$.

\subsection{Existence of approximations in (3)}

Existence of some $x_{i+1} \in \mathbb{R}^{n}$ for any $x_{i} \in \mathbb{R}^{n}$ in the explicit case (2) is straightforward, but it is not the case of (3). According to the result of Corollary 6 , it is enough to find the conditions of existence of $x_{i+1}$ for all $x_{i} \in S_{r}(1)$ in (3). Note that in a general case, it is difficult to provide some simple conditions for existence and uniqueness of $x_{i+1}$ in the equation (3) for any $x_{0} \in S_{r}(1)$, but homogeneity may further simplify the solution under additional mild restrictions on $f$.

Proposition 11 Let $f$ be $\mathbf{r}$-homogeneous of degree $\nu \in$ $\mathbb{R}$ and continuous on $S_{r}(1)$. Then there is $h_{0}>0$ such that for all $h \in\left(0, h_{0}\right)$ the equation (3) for any $x_{i} \in \mathbb{R}^{n}$ has a solution $x_{i+1} \in \mathbb{R}^{n}$.

PROOF. The proof is given in [20].

Thus, for the proposed implicit Euler method with statedependent discretization step (3), the global existence of solutions may be guaranteed by selecting $h$ sufficiently small provided that $f$ is homogeneous and continuous.

\subsection{Convergence of the implicit Euler scheme (3)}

Similar to (2) results can be obtained for (3) and even with some improvement under additional restrictions. Note that in this subsection we assume that the sequences $\left\{x_{i}\right\}_{i=0}^{\infty}$ exist in (3) for all $x_{0} \in \mathbb{R}^{n}$, e.g. the conditions of Proposition 11 are verified.

Theorem 12 Let Assumption 1 be satisfied, then there exists $h_{0}>0$ such that for any discretization step $h \in$ $\left(0, h_{0}\right]$ the sequences $\left\{x_{i}\right\}_{i=0}^{\infty}$ obtained by (3) for any initial state $x_{0} \in \mathbb{R}^{n}$ and the step $h$ possess:

(a) $\sup _{i=0,1, \ldots}\left\|x_{i}\right\|_{r}<\gamma\left\|x_{0}\right\|_{r}$ for some $\gamma \in(0,+\infty)$; (b) $\lim _{i \rightarrow+\infty}\left\|x_{i}\right\|_{r}=0$;

(c) for $\nu=0$ the sequence $\left\{x_{i}\right\}_{i=0}^{\infty}$ has an exponential convergence rate, for $\nu<0$ for any $x_{0} \in \mathbb{R}^{n}$ the time of convergence to the origin $t_{+\infty}^{x_{0}}=\lim _{i \rightarrow+\infty} t_{i}$ is finite, and for $\nu>0$ the time of convergence from any $x_{0} \in \mathbb{R}^{n}$ to $B_{r}(1)$ is also finite independently of $x_{0}$.

If the matrix $\frac{\partial^{2} V(\xi)}{\partial \xi^{2}} \geq 0$ for all $\xi \in \mathbb{R}^{n}$, where $V: \mathbb{R}^{n} \rightarrow$ $\mathbb{R}_{+}$is a $\mathbf{r}$-homogeneous Lyapunov function satisfying (7), then $h_{0}>0$ can be selected arbitrary.

PROOF. The proof is given in [20].

The requirement on nonnegative definiteness of the second derivative of $V$ is related with the condition of convexity level set of $V$ imposed in [18].

\section{$5 \quad$ Absolute and Relative Errors of Discretized Homogeneous Systems}

Conventional characteristics of the discretization accuracy are studied in this section for the proposed algorithms (2) or (3) and for homogeneous systems. To this end denote $\Xi_{h}\left(x_{0}\right)=X\left(h\left\|x_{0}\right\|_{r}^{-\nu}, x_{0}\right)$ as the value of the solution of (1) with the initial condition $x_{0} \in \mathbb{R}^{n}$ evaluated in (2) or (3) after one iteration with the discretization step $h>0$ (at $t_{1}=h\left\|x_{0}\right\|_{r}^{-\nu}$ with $t_{0}=0$ ). Denote by $\hat{\Xi}_{h}\left(x_{0}\right)$ the estimated value derived by (2) or (3) for the same $x_{0}$ and $h>0$ (note that $\hat{\Xi}_{h}\left(x_{0}\right)=x_{0}+\frac{h}{\left\|x_{0}\right\|_{r}^{\nu}} f\left(x_{0}\right)$ in the case of $(2))$, then [15]:

- the absolute error is the magnitude of the difference between the exact value and its approximation:

$$
\begin{aligned}
& \Delta^{h}(x)=\left\|\Xi_{h}(x)-\hat{\Xi}_{h}(x)\right\|, \\
& \Delta_{r}^{h}(x)=\left\|\Xi_{h}(x)-\hat{\Xi}_{h}(x)\right\|_{r} ;
\end{aligned}
$$

- the relative error expresses how large the absolute error is compared with the exact value:

$$
\delta^{h}(x)=\frac{\Delta^{h}(x)}{\left\|\Xi_{h}(x)\right\|}, \quad \delta_{r}^{h}(x)=\frac{\Delta_{r}^{h}(x)}{\left\|\Xi_{h}(x)\right\|_{r}} .
$$

The errors are given for two different norms, the conventional one $\|\cdot\|$ and the homogeneous norm $\|\cdot\|_{r}$, the former one is used habitually for evaluation of a discretization method precision, while the latter norm suits better for analysis of homogeneous systems. Equivalence of these norms has been shown in [18], that is why in this note we will study only $\Delta_{r}^{h}\left(x_{i}\right)$ and $\delta_{r}^{h}\left(x_{i}\right)$.

These error functions admit the following useful properties: 
Theorem 13 Let the system (1) be $\mathbf{r}$-homogeneous of degree $\nu \in \mathbb{R}$ and $\hat{\Xi}_{h}(x)$ be calculated by the explicit (2) or implicit (3) Euler scheme for $x \in \mathbb{R}^{n}$ and $h>0$. Then the functions $\Delta_{r}^{h}(x)$ and $\delta_{r}^{h}(x)$ are $\mathbf{r}$-homogeneous of degree 1 and 0 , respectively.

PROOF. First of all note that for a homogeneous system (1) [6]:

$$
X\left(t, \Lambda_{r}(\lambda) x\right)=\Lambda_{r}(\lambda) X\left(\lambda^{\nu} t, x\right) \quad \forall t \geq 0, \forall x \in \mathbb{R}^{n}
$$

for any $\lambda>0$, then

$$
\begin{aligned}
\Xi_{h}\left(\Lambda_{r}(\lambda) x\right) & =X\left(h\left\|\Lambda_{r}(\lambda) x\right\|_{r}^{-\nu}, \Lambda_{r}(\lambda) x\right) \\
& =X\left(\lambda^{-\nu} h\|x\|_{r}^{-\nu}, \Lambda_{r}(\lambda) x\right) \\
& =\Lambda_{r}(\lambda) X\left(h\|x\|_{r}^{-\nu}, x\right)=\Lambda_{r}(\lambda) \Xi_{h}(x),
\end{aligned}
$$

hence $\Xi_{h}$ is $\mathbf{r}$-homogeneous vector field of degree 0 . According to Proposition (5) the vector fields $\hat{\Xi}_{h}(x)$ are also $\mathbf{r}$-homogeneous of degree 0 in (2) or (3).

Consider the case of the explicit Euler scheme (2), then

$$
\begin{aligned}
\Delta_{r}^{h}(x) & =\left\|\Xi_{h}(x)-\hat{\Xi}_{h}(x)\right\|_{r} \\
& =\left\|\Xi_{h}(x)-x-h\right\| x\left\|_{r}^{-\nu} f(x)\right\|_{r}
\end{aligned}
$$

and for any $\lambda>0$ we obtain:

$$
\begin{aligned}
\Delta_{r}^{h}\left(\Lambda_{r}(\lambda) x\right)= & \| \Xi_{h}\left(\Lambda_{r}(\lambda) x\right)-\Lambda_{r}(\lambda) x \\
& -h\left\|\Lambda_{r}(\lambda) x\right\|_{r}^{-\nu} f\left(\Lambda_{r}(\lambda) x\right) \|_{r} \\
= & \lambda\left\|\Xi_{h}(x)-x-h\right\| x\left\|_{r}^{-\nu} f(x)\right\|_{r}=\lambda \Delta_{r}^{h}(x),
\end{aligned}
$$

which implies that the error function $\Delta_{r}^{h}(x)$ is $\mathbf{r}$-homogeneous function of degree 1. Similarly,

$$
\delta_{r}^{h}\left(\Lambda_{r}(\lambda) x\right)=\frac{\Delta_{r}^{h}\left(\Lambda_{r}(\lambda) x\right)}{\left\|\Xi_{h}\left(\Lambda_{r}(\lambda) x\right)\right\|_{r}}=\frac{\Delta_{r}^{h}(x)}{\left\|\Xi_{h}(x)\right\|_{r}}=\delta_{r}^{h}(x)
$$

for any $\lambda>0$ and the error function $\delta_{r}^{h}(x)$ is $\mathbf{r}$-homogeneous function of degree 0 .

For the case of the implicit Euler method (3) we have:

$$
\begin{aligned}
\Delta_{r}^{h}(x) & =\left\|\Xi_{h}(x)-\hat{\Xi}_{h}(x)\right\|_{r} \\
& =\left\|\Xi_{h}\left(\hat{\Xi}_{h}(x)-h\left\|\hat{\Xi}_{h}(x)\right\|_{r}^{-\nu} f\left(\hat{\Xi}_{h}(x)\right)\right)-\hat{\Xi}_{h}(x)\right\|_{r}
\end{aligned}
$$

and repeating the same arguments (as it has been shown above, $\Xi_{h}(x)$ and $\hat{\Xi}_{h}(x)$ are $\mathbf{r}$-homogeneous of degree 0$)$ the following facts can be substantiated:

$$
\Delta_{r}^{h}\left(\Lambda_{r}(\lambda) x\right)=\lambda \Delta_{r}^{h}(x), \delta_{r}^{h}\left(\Lambda_{r}(\lambda) x\right)=\delta_{r}^{h}(x) .
$$

Any homogeneous function of degree 0 is globally bounded (it may be discontinuous) if its maximal amplitude is finite being evaluated on $S_{r}(1)$. Therefore, if for any initial conditions $x \in S_{r}(1)$ the error $\delta_{r}^{h}(x)$ stays sufficiently small for a reasonable selection of $h$ (i.e. the one step error of usual Euler discretization approaches is small on the sphere), then the explicit (2) and the implicit (3) Euler schemes provide a uniformly bounded relative error $\delta_{r}$ globally. Boundedness of $\delta_{r}$ implies that the difference between $\Xi_{h}(x)$ and $\hat{\Xi}_{h}(x)$ stays of the order $\Xi_{h}(x)$ (roughly speaking proportional to $x$ ). Indeed, assume that $f$ is continuously differentiable on $S_{r}(1)$. In this scenario, the second derivative of the solution $X\left(t, x_{0}\right)$ exists and continuous, and for all $x_{0} \in S_{r}(1)$ :

$$
\begin{gathered}
\Xi_{h}\left(x_{0}\right)=X\left(h\left\|x_{0}\right\|_{r}^{-\nu}, x_{0}\right)=x_{0}+h \dot{X}\left(0, x_{0}\right)+\frac{h^{2}}{2} \ddot{X}\left(\theta, x_{0}\right) \\
=x_{0}+h f\left(x_{0}\right)+\frac{h^{2}}{2} \ddot{X}\left(\theta, x_{0}\right),
\end{gathered}
$$

where $\theta \in[0, h]$ and the Lagrange reminder of Taylor series expansion was used. Then for $x_{0} \in S_{r}(1)$ and the explicit discretization algorithm (2):

$$
\begin{gathered}
\Delta_{r}^{h}\left(x_{0}\right)=\left\|\Xi_{h}\left(x_{0}\right)-\hat{\Xi}_{h}\left(x_{0}\right)\right\|_{r} \\
=\left\|x_{0}+h f\left(x_{0}\right)+\frac{h^{2}}{2} \ddot{X}\left(\theta, x_{0}\right)-x_{0}-h f\left(x_{0}\right)\right\|_{r} \\
=\frac{h^{2}}{2}\left\|\ddot{X}\left(\theta, x_{0}\right)\right\|_{r}
\end{gathered}
$$

for $h \leq 1$ and $r_{\max }=1$ (always can be assumed without loosing generality). By differentiability of $f$, there exists a constant $\varsigma>0$ such that

$$
\sup _{\theta \in[0,1], x_{0} \in S_{r}(1)}\left\|\ddot{X}\left(\theta, x_{0}\right)\right\|_{r} \leq \varsigma,
$$

hence,

$$
\Delta_{r}^{h}\left(x_{0}\right) \leq \frac{\varsigma}{2} h^{2}
$$

for all $x_{0} \in S_{r}(1)$ and $h \in(0,1]$. The quadratic in $h$ convergence of the absolute error $\Delta_{r}^{h}\left(x_{0}\right)$ (the same can be shown for the relative error $\left.\delta_{r}^{h}\left(x_{0}\right)\right)$ implies approaching of the real solution $X\left(h, x_{0}\right)$ by its approximation given in (2) [15].

Remark 14 Note that the time step in (2) and (3) is state- and degree-dependent. In particular, if $\left\|x_{i}\right\|_{r} \gg$ 1 (it is sufficiently big) and $\nu<0$, then $t_{i+1}-t_{i}=$ $\left\|x_{i}\right\|_{r}^{-\nu} h \gg h$ in (2) and the time step can be too large, which is also related with the obtained accuracy estimates in Theorem 13. It is worth stressing that anyway the convergence of these algorithms is not influenced, and this observation deals only with the sampling of discretization. Therefore, for big amplitudes of $x_{i}$ in the case of $\nu<0$ it is admissible to use the conventional Euler methods (without a scaling of the time discretization step), which 
may provide a reasonable accuracy of approximation of the solutions under a more regular sampling [18,30].

\section{Robustness with respect to external inputs}

Let us consider a version of the system (1) extended by external inputs:

$$
\dot{x}(t)=F(x(t), d(t)), t \geq 0,
$$

with $F: \mathbb{R}^{n+m} \rightarrow \mathbb{R}^{n}$ and $F(x, 0)=f(x)$, where $d:$ $\mathbb{R}_{+} \rightarrow \mathbb{R}^{m}$ is a measurable and essentially bounded function of time. For $x_{0} \in \mathbb{R}^{n}$ and an input $d: \mathbb{R}_{+} \rightarrow \mathbb{R}^{m}$ denote a corresponding solution of (8) as $X\left(t, x_{0}, d\right)$. The following hypothesis will be imposed on (8):

Assumption 2 There exist $\tilde{r}_{j}>0, j=\overline{1, m}$ and $\nu \in \mathbb{R}$ such that

$$
F\left(\Lambda_{r}(\lambda) x, \Lambda_{\tilde{r}}(\lambda) d\right)=\lambda^{\nu} \Lambda_{r}(\lambda) F(x, d)
$$

for all $x \in \mathbb{R}^{n}, d \in \mathbb{R}^{m}$ and $\lambda>0$, and there exists $\sigma \in \mathcal{K}_{\infty}$ such that

$$
\sup _{y \in S_{r}(1)}\|F(y, d)-F(y, 0)\| \leq \sigma\left(\|d\|_{\tilde{r}}\right)
$$

for all $d \in \mathbb{R}^{m}$

If Assumption 1 is also satisfied, since $F(x, 0)=f(x)$, the degrees $\nu$ in these assumptions coincide. Moreover, if assumptions 1 and 2 are both verified, then the system (8) possesses the input-to-state stability (ISS) property with respect to $d[9]$, [10], and the zone of asymptotic convergence (the asymptotic gain) can be evaluated as

$$
\|x\|_{r} \leq\left(\frac{c_{2}}{c_{1}}\right)^{\frac{1}{\mu}} \frac{\|d\|_{\tilde{r}, \infty}}{\sigma^{-1}\left(\frac{a}{2 b}\right)},
$$

where constants $\mu, a, b, c_{1}$ and $c_{2}$ are introduced in (7),

$$
\|d\|_{\tilde{r}, \infty}=\operatorname{ess} \sup _{t \geq 0}\|d(t)\|_{\tilde{r}}
$$

Let us show that (2) and (3) preserve the same stability performance for (8). Note that being applied to (8) these algorithms can be formulated as follows:

$$
\begin{aligned}
x_{i+1} & =x_{i}+\frac{h}{\left\|x_{i}\right\|_{r}^{\nu}} F\left(x_{i}, d_{i}\right), \\
t_{i+1} & =t_{i}+\frac{h}{\left\|x_{i}\right\|_{r}^{\nu}}
\end{aligned}
$$

or

$$
\begin{aligned}
x_{i+1} & =x_{i}+\frac{h}{\left\|x_{i+1}\right\|_{r}^{\nu}} F\left(x_{i+1}, d_{i+1}\right), \\
t_{i+1} & =t_{i}+\frac{h}{\left\|x_{i+1}\right\|_{r}^{\nu}}
\end{aligned}
$$

for $i=0,1, \ldots$, respectively, where as before $x_{i}$ is an estimate of $X\left(t_{i}, x_{0}, d\right)$ and $d_{i}=d\left(t_{i}\right)$.

Theorem 15 Let assumptions 1 and 2 be satisfied, then there exists $h_{0}>0$ such that for any discretization step $h \in\left(0, h_{0}\right]$ the discrete-time systems (9) or (10) are $I S S^{1}$, and for any initial state $x_{0} \in \mathbb{R}^{n}$ and any bounded input $d: \mathbb{R}_{+} \rightarrow \mathbb{R}^{m}$ the corresponding sequences $\left\{x_{i}\right\}_{i=0}^{\infty}$ enter in the set where

$$
\left\|x_{i}\right\|_{r} \leq\left(\frac{c_{2}}{c_{1}}\right)^{\frac{1}{\mu}} \frac{\|d\|_{\tilde{r}, \infty}}{\sigma^{-1}\left(\frac{a}{2 b}\right)} .
$$

PROOF. If Assumption 1 is verified, then there exists a Lyapunov function $V(x)$ for the system (1) that satisfies the conditions (7).

Let us check the increment of this Lyapunov function $V(x)$ on the sequences produced by $(9)$ (i.e. let us verify that $V(x)$ is an ISS Lyapunov function for (9) [26]):

$$
\begin{aligned}
V\left(x_{i+1}\right) & -V\left(x_{i}\right)=V\left(x_{i}+h\left\|x_{i}\right\|_{r}^{-\nu} F\left(x_{i}, d_{i}\right)\right)-V\left(x_{i}\right) \\
& =\left\|x_{i}\right\|_{r}^{\mu}\left[V\left(y_{i}+h F\left(y_{i}, \delta_{i}\right)\right)-V\left(y_{i}\right)\right],
\end{aligned}
$$

where $y_{i} \in S_{r}(1)$ such that $x_{i}=\Lambda_{r}\left(\left\|x_{i}\right\|_{r}\right) y_{i}, d_{i}=$ $\Lambda_{\tilde{r}}\left(\left\|x_{i}\right\|_{r}\right) \delta_{i}$ for some $\delta_{i} \in \mathbb{R}^{m}$, and the first property from Assumption 2 has been used. Next, similarly to the proof of Theorem 9 , for some $\theta \in[0,1]$ we obtain:

$$
\begin{gathered}
V\left(y_{i}+h F\left(y_{i}, \delta_{i}\right)\right)=V\left(y_{i}\right)+\left.\frac{\partial V(\xi)}{\partial \xi}\right|_{\xi=y_{i}} h F\left(y_{i}, \delta_{i}\right) \\
+\left.\frac{h^{2}}{2} F^{\top}\left(y_{i}, \delta_{i}\right) \frac{\partial^{2} V(\xi)}{\partial \xi^{2}}\right|_{\xi=y_{i}+\theta h F\left(y_{i}, \delta_{i}\right)} F\left(y_{i}, \delta_{i}\right) \\
=V\left(y_{i}\right)+\left.\frac{\partial V(\xi)}{\partial \xi}\right|_{\xi=y_{i}} h f\left(y_{i}\right) \\
+\left.\frac{\partial V(\xi)}{\partial \xi}\right|_{\xi=y_{i}} h\left[F\left(y_{i}, \delta_{i}\right)-F\left(y_{i}, 0\right)\right] \\
+\left.\frac{h^{2}}{2} F^{\top}\left(y_{i}, \delta_{i}\right) \frac{\partial^{2} V(\xi)}{\partial \xi^{2}}\right|_{\xi=y_{i}+\theta h F\left(y_{i}, \delta_{i}\right)} F\left(y_{i}, \delta_{i}\right) \\
\leq V\left(y_{i}\right)-h a+h b \sigma\left(\left\|\delta_{i}\right\|_{\tilde{r}}\right) \\
+\left.\frac{h^{2}}{2} F^{\top}\left(y_{i}, \delta_{i}\right) \frac{\partial^{2} V(\xi)}{\partial \xi^{2}}\right|_{\xi=y_{i}+\theta h F\left(y_{i}, \delta_{i}\right)} F\left(y_{i}, \delta_{i}\right)
\end{gathered}
$$

1 See [26] for the definition of this property and also for its equivalent Lyapunov characterizations. 
where the estimates from (7) and the second property from Assumption 2 are used on the last step. Denote

$$
\begin{gathered}
w=\sup _{y \in S_{r}(1)\|\delta\|_{\tilde{r}} \leq \sigma^{-1}\left(\frac{a}{2 b}\right)} \sup _{\left.\sup _{\iota \in[0,1]} F^{\top}(y, \delta) \frac{\partial^{2} V(\xi)}{\partial \xi^{2}}\right|_{\xi=y+\iota F(y, \delta)} F(y, \delta),}
\end{gathered}
$$

obviously $w \in(0,+\infty)$ (recall that $\sup _{y \in S_{r}(1)}\|f(y)\|<$ $+\infty$ and $\|F(y, \delta)\| \leq\|F(y, 0)\|+\sigma\left(\|\delta\|_{\tilde{r}}\right)$ for all $y \in S_{r}$ and $\delta \in \mathbb{R}^{m}$ by Assumption 2), and assume that

$$
\left\|\delta_{i}\right\|_{\tilde{r}} \leq \sigma^{-1}\left(\frac{a}{2 b}\right)
$$

then

$$
\begin{aligned}
& V\left(y_{i}+h F\left(y_{i}, \delta_{i}\right)\right)- V\left(y_{i}\right) \leq-h a+h b \sigma\left(\left\|\delta_{i}\right\|_{\tilde{r}}\right)+\frac{h^{2}}{2} w \\
& \leq-h \frac{a}{2}+\frac{h^{2}}{2} w
\end{aligned}
$$

provided that $h \leq 1$. In addition, if $h \in\left(0, h_{0}\right]$ with $h_{0}=\min \left\{1, \frac{a}{2 w}\right\}$, then

$$
V\left(x_{i+1}\right)-V\left(x_{i}\right) \leq-h \frac{a}{4}\left\|x_{i}\right\|_{r}^{\mu}
$$

under the restriction (11), which can be equivalently rewritten as $\left\|x_{i}\right\|_{r} \geq \frac{\|d\|_{\tilde{r}, \infty}}{\sigma^{-1}\left(\frac{a}{2 b}\right)}$ or

$$
V\left(x_{i}\right) \geq c_{2}\left(\frac{\|d\|_{\tilde{r}, \infty}}{\sigma^{-1}\left(\frac{a}{2 b}\right)}\right)^{\mu} .
$$

Therefore, the system (9) is ISS [26], and any sequence $\left\{x_{i}\right\}_{i=0}^{\infty}$ generated by (9) reaches for the zone

$$
c_{1}\left\|x_{i}\right\|_{r}^{\mu} \leq V\left(x_{i}\right) \leq c_{2}\left(\frac{\|d\|_{\tilde{r}, \infty}}{\sigma^{-1}\left(\frac{a}{2 b}\right)}\right)^{\mu},
$$

which is desired.

For the system (3) the analysis is similar:

$$
\begin{gathered}
V\left(x_{i+1}\right)-V\left(x_{i}\right)=V\left(x_{i+1}\right)-V\left(x_{i+1}-h F\left(x_{i+1}, d_{i+1}\right)\right) \\
\quad=\left\|x_{i+1}\right\|_{r}^{\mu}\left[V\left(y_{i+1}\right)-V\left(y_{i+1}-h F\left(y_{i+1}, \delta_{i+1}\right)\right)\right]
\end{gathered}
$$

where $x_{i+1}=\Lambda_{r}\left(\left\|x_{i+1}\right\|_{r}\right) y_{i+1}$ for $y_{i+1} \in S_{r}(1)$ and $d_{i+1}=\Lambda_{\tilde{r}}\left(\left\|x_{i+1}\right\|_{r}\right) \delta_{i+1}$ for some $\delta_{i+1} \in \mathbb{R}^{m}$. Next, all the rest steps coincide.

Therefore, the schemes (2) and (3) (or (9) and (10)) for approximation of solutions of a homogeneous system (8) keep unchanged the convergence rates and stability margins in the presence of perturbations.
Remark 16 Note that for an input $d(t) \neq 0$ the corresponding trajectory may visit the origin, but does not stay there due to disturbance presence. In such a case the algorithms (9) and (10) will decrease to zero the amplitude of the time step for the systems with negative degree or augment it till infinity for the systems with positive degree following their construction. In order to avoid this issue, the upper and lower limits $0<\underline{h}<\bar{h}<+\infty$ on $h\left\|x_{i}\right\|_{r}^{-\nu}$ or $h\left\|x_{i+1}\right\|_{r}^{-\nu}$ must be imposed in (9) and (10), respectively.

\section{$7 \quad$ Example}

Consider the super-twisting benchmark system:

$\dot{x}_{1}=-k_{1}\left|x_{1}\right|^{0.5} \operatorname{sign}\left(x_{1}\right)+x_{2}+d$,

$\dot{x}_{2}=-k_{2} \operatorname{sign}\left(x_{1}\right)+f$,

where $x=\left[\begin{array}{ll}x_{1} & x_{2}\end{array}\right]^{\top} \in \mathbb{R}^{2}$ is the state vector, $k_{1}>0$ and $k_{2}>0$ are the system parameters; $d \in \mathbb{R}$ is the external disturbance and $f \in \mathbb{R}$ is the matched perturbation that has a known upper bound with $k_{2}>\sup _{t>0}|f(t)|$; $\nu=-0.5$ is the homogeneity degree for $\mathbf{r}=\left[\begin{array}{ll}1 & 0.5\end{array}\right]^{\top}$. The signal $f$ can be embedded in the system equations by considering instead a differential inclusion, and it is easy to check that then the super-twisting algorithm is an example of the system (8), which verifies assumptions 1 and 2 (for $\tilde{\mathbf{r}}=0.5$ and $\sigma(s)=s$ ). Since it is a discontinuous system, then we are going to compare for this model the explicit method (9) and the same approach without state-dependent scaling:

$$
x_{i+1}=x_{i}+h F\left(x_{i}, d_{i}\right), t_{i}=i h
$$

for $i=0,1, \ldots$, which was previously studied in many works and it is recommended for the use in the sliding mode control community $[28,18,30,29]$ (a more sophisticated solution is given in [32]).

For simulations $k_{1}=8$ and $k_{2}=5$ are selected with

$$
f(t)=\frac{k_{2}}{3}\left(1+\sin \left(2 t^{2}\right)\right)
$$

and the run of the algorithms (9) (for $i=0, \ldots, N$ with the maximum number of iterations $N=10^{4}$ ) and (12) have been performed for the same initial condition $x_{0}=\left[\begin{array}{ll}10 & 10\end{array}\right]^{\top}$ with the discretization step $h=10^{-2}$ and $\bar{h}=\underline{h}^{-1}=10^{10}$ from Remark 16 . The results of simulation for the case $d(t)=0$ are presented in Fig. 1, where $\|x(t)\|_{r}$ is plotted in a logarithmic scale, the result of (9) corresponds to the blue line, the result of (12) is shown by the red one. As we can conclude from these simulations, the method (9) outperforms (12) since the former is finite-time converging, while the latter one cannot converge to the origin. It is important to highlight that these discretization algorithms have a similar computational 


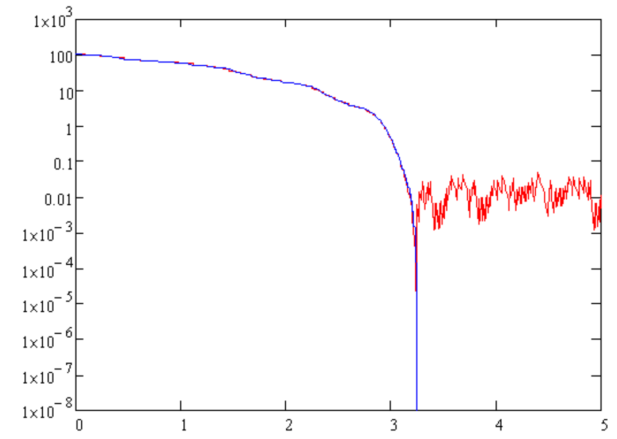

Fig. 1. The results of simulation for $d(t)=0$

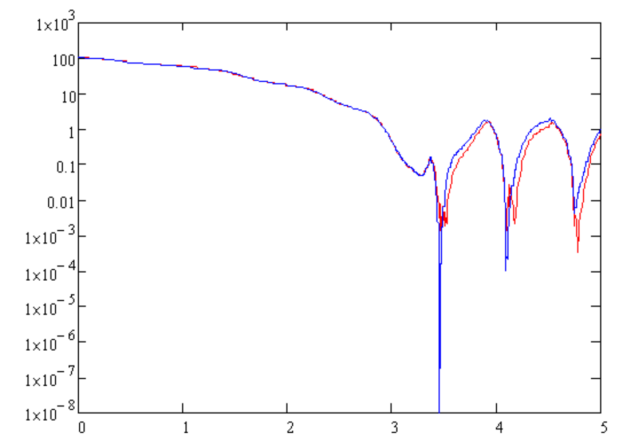

Fig. 2. The results of simulation for $d(t)=1+\cos (10 t)$

complexity (comparing with (12), in (9) it is necessary to additionally compute the value of the norm $\left\|x_{i}\right\|_{r}^{\nu}$, which only needs the power and the addition operations). The results of simulation for $d(t)=1+\cos (10 t)$ are plotted in Fig. 2, where (9) is approaching the origin much closer, while (12) generates bounded trajectories loosing the precision in a vicinity of zero. All these results confirm the theoretical finding of the paper.

\section{Conclusions}

In this work a series of results has been established devoted to application of the explicit and implicit Euler methods with state-dependent scaling of the discretization step for discrete-time approximation of solutions of homogeneous systems. The main contributions can be summarized as follows:

- The obtained approximations can be made globally converging for asymptotically stable homogeneous systems provided that the discretization step is selected sufficiently small, in addition they preserve finite-time or fixed-time rates of convergence in infinite number of iterations (theorems 9 and 12). The maximal admissible discretization step can be evaluated on the sphere (Remark 10).

- For any value of the discretization step, the implicit and explicit Euler methods provide a good approximation of the system solutions (Theorem 13).

- Both approaches, (2) and (3) ((9) and (10)), preserve the input-to-state stability of the homogeneous dynamics (8) with the same asymptotic gain (Theorem $15)$.

\section{References}

[1] V. Acary and B. Brogliato. Implicit euler numerical simulations of sliding mode systems. System and Control Letters, 59(5):284-293, 2010.

[2] A.Yu. Aleksandrov. On the asymptotical stability of solutions of nonstationary differential equation systems with homogeneous right hand sides. Dokl. Akad. Nauk Rossii, 349(3):295-296, 1996. in Russian.

[3] A.Yu. Aleksandrov and A.P. Zhabko. Preservation of stability under discretization of systems of ordinary differential equations. Siberian Math. J., 51(3):383-395, 2010.

[4] M.B. Allen and E.L. Isaacson. Numerical Analysis for Applied Science. A Wiley-Interscience publication. Wiley, 1998.

[5] V. Andrieu, L. Praly, and A. Astolfi. Homogeneous Approximation, Recursive Observer Design, and Output Feedback. SIAM J. Control Optimization, 47(4):1814-1850, 2008.

[6] A. Bacciotti and L. Rosier. Lyapunov Functions and Stability in Control Theory. Springer, Berlin, 2nd edition, 2005.

[7] E. Bernuau, D. Efimov, W. Perruquetti, and A. Polyakov. On homogeneity and its application in sliding mode. Int. J. Franklin Institute, 351(4):1866-1901, 2014.

[8] E. Bernuau, A. Polyakov, D. Efimov, and W. Perruquetti. On extension of homogeneity notion for differential inclusions. In Proceeding of the European Control Conference, pages 22042209, Zurich, 2013.

[9] E. Bernuau, A. Polyakov, D. Efimov, and W. Perruquetti. Verification of ISS, iISS and IOSS properties applying weighted homogeneity. Systems \& Control Letters, 62:11591167, 2013.

[10] Emmanuel Bernuau, Denis Efimov, and Wilfrid Perruquetti. Robustness of Homogeneous and Homogeneizable Differential Inclusions, pages 39-56. Springer International Publishing, Cham, 2018.

[11] S. P. Bhat and D. S. Bernstein. Geometric homogeneity with applications to finite-time stability. Mathematics of Control, Signals and Systems, 17:101-127, 2005.

[12] B. Brogliato and A. Polyakov. Globally stable implicit euler time-discretization of a nonlinear single-input sliding-mode control system. In IEEE CDC, pages 5426-5431, 2015.

[13] John C. Butcher. Numerical Methods for Ordinary Differential Equations. John Wiley \& Sons, New York, 2nd edition, 2008.

[14] E. Cruz-Zavala, J.A. Moreno, and L.M. Fridman. Uniform robust exact differentiator. IEEE Transactions on Automatic Control, 56(11):2727-2733, 2011.

[15] G. Dahlquist and A. Björck. Numerical methods in scientific computing. SIAM, 2008.

[16] Germund G. Dahlquist. A special stability problem for linear multistep methods. BIT Numerical Mathematics, 3(1):2743, 1963.

[17] D. Efimov, A. Polyakov, and A. Aleksandrov. On statedependent discretization of stable homogeneous systems. In Proc. 57th IEEE Conference on Decision and Control (CDC), Fontainebleau (FL), 2018. 
[18] D. Efimov, A. Polyakov, A. Levant, and W. Perruquetti. Realization and discretization of asymptotically stable homogeneous systems. IEEE Transactions on Automatic Control, 62(11):5962-5969, 2017.

[19] D. Efimov, A. Polyakov, W. Perruquetti, and J.-P. Richard. Weighted homogeneity for time-delay systems: Finite-time and independent of delay stability. IEEE Transactions on Automatic Control, 61(1):210-215, 2016.

[20] Denis Efimov, Andrey Polyakov, and Alexander Aleksandrov. Proofs for "Discretization of Homogeneous Systems Using Euler Method with a State-Dependent Step". https://hal.inria.fr/hal-02164050, June 2019.

[21] A.F. Filippov. Differential Equations with Discontinuous Righthand Sides. Kluwer Academic Publishers, 1988.

[22] C. Gonzalez, A. Ostermann, C. Palencia, and M. Thalhammer. Backward euler discretization of fully nonlinear parabolic problems. Mathematics of Computation, 71(237):125-145, 2002.

[23] Lars Grüne. Homogeneous state feedback stabilization of homogeneous systems. SIAM J. Control Optimization, 38(4):1288-1314, 2000.

[24] Yiguang Hong. $\mathrm{H}_{\infty}$ control, stabilization, and input-output stability of nonlinear systems with homogeneous properties. Automatica, 37(7):819-829, 2001.

[25] O. Huber, V. Acary, and B. Brogliato. Lyapunov stability and performance analysis of the implicit discrete sliding mode control. IEEE Transactions on Automatic Control, 61(10):3016-3030, 2016.

[26] Zhong-Ping Jiang and Yuan Wang. Input-to-state stability for discrete-time nonlinear systems. Automatica, 37:857-869, 2001.

[27] H. K. Khalil. Nonlinear Systems. NJ 07458. Prentice-Hall, Upper Saddle River, 1996.

[28] A. Levant. Homogeneity approach to high-order sliding mode design. Automatica, 41(5):823-830, 2005.

[29] A. Levant. On fixed and finite time stability in sliding mode control. In IEEE 52nd Annual Conference on Decision and Control (CDC), pages 4260-4265, 2013.

[30] A. Levant, D. Efimov, A. Polyakov, and W. Perruquetti. Stability and robustness of homogeneous differential inclusions. In Proc. IEEE CDC, 2016.

[31] J. Li and C. Qian. Global finite-time stabilization by dynamic output feedback for a class of continuous nonlinear systems. IEEE Transactions on Automatic Control, 51(5):879-884, 2006.

[32] Miki Livne and Arie Levant. Proper discretization of homogeneous differentiators. Automatica, 50(8):2007-2014, 2014.

[33] B. Merlet and M. Pierre. Convergence to equilibrium for the backward euler scheme and applications. Communications on Pure and Applied Analysis, 9(3):685-702, 2010.

[34] F. A. Miranda-Villatoro, B. Brogliato, and F. Castanos. Multivalued robust tracking control of lagrange systems: Continuous and discrete-time algorithms. IEEE Transactions on Automatic Control, 62(9):4436-4450, 2017.

[35] E. Moulay and W. Perruquetti. Finite time stability of differential inclusions. IMA J. Math. Control Inform, 22(4):465-475, 2005.

[36] J. Peuteman and D. Aeyels. Averaging results and the study of uniform asymptotic stability of homogeneous differential equations that are not fast time-varying. SIAM J. Control Optim, 37(4):997-1010, 1999.
[37] A. Polyakov. Nonlinear feedback design for fixed-time stabilization of linear control systems. IEEE Transactions on Automatic Control, 57(8):2106-2110, 2012.

[38] A. Polyakov, D. Efimov, and W. Perruquetti. Finite-time and fixed-time stabilization: Implicit lyapunov function approach. Automatica, 51:332-340, 2015.

[39] L. Rosier. Homogeneous Lyapunov function for homogeneous continuous vector field. Systems \& Control Letters, 19:467473, 1992.

[40] E. Roxin. On finite stability in control systems. Rendiconti del Circolo Matematico di Palermo, 15:273-283, 1966.

[41] Yanjun Shen and Xiaohua Xia. Semi-global finite-time observers for nonlinear systems. Automatica, 44(12):31523156, 2008.

[42] Hong Yiguang, Jiang Zhong-Ping, and Feng Gang. Finitetime input-to-state stability and applications to finite-time control design. SIAM J. Control Optim., 48(7):4395-4418, 2010.

[43] K. Zimenko, D. Efimov, A. Polyakov, and W. Perruquetti. A note on delay robustness for homogeneous systems with negative degree. Automatica, 79(5):178-184, 2017.

[44] V.I. Zubov. On systems of ordinary differential equations with generalized homogenous right-hand sides. Izvestia vuzov. Mathematica., 1:80-88, 1958 (in Russian). 